\title{
Avaliação radiológica e funcional dos pacientes com fratura da coluna cervical subaxial
}

\author{
Functional and radiological assessment of patients with subaxial \\ cervical fractures \\ La evaluación radiológica y funcional de los pacientes con fracturas \\ de la columna cervical subaxial
}

\author{
Fabiano Ricardo de Tavares Canto' \\ Paulo Roberto Santos Neto ${ }^{2}$ \\ Ilton José Carrilho de Castro' \\ Roberto Sérgio de Tavares Canto ${ }^{3}$ \\ Hector Abreu dos Santos ${ }^{4}$ \\ Marcus Vinicius Martins do Nascimento ${ }^{4}$
}

\section{RESUMO}

Objetivo: Avaliar 17 pacientes portadores de fratura luxação da coluna cervical (C3 a C7), que foram tratados em um período de dois anos. Os pacientes foram avaliados com parâmetros radiológicos, clínicos e funcionais. Métodos: A indicação do tratamento utilizado em cada paciente foi baseada em seu quadro clínico e neurológico. Todos os pacientes foram tratados pela mesma técnica cirúrgica. $\mathrm{O}$ seguimento pósoperatório mínimo foi de seis meses. Resultados: Dez pacientes não apresentavam nenhum comprometimento neurológico e sete pacientes apresentavam algum grau de acometimento neurológico. Na avaliação radiográfica do seguimento ambulatorial, todos os pacientes apresentaram sinais de consolidação da artrodese. Todos ficaram relacionados entre os níveis excelente e regular, de acordo com os critérios de Odom. Treze pacientes apresentaram $20 \%$ de limitação pelo questionário Neck Pain Disability Index. Encontrou-

\begin{abstract}
Objective: To evaluate 17 patients with cervical (C3-C7) traumatic injuries treated in a period of two years. Patients were evaluated based on clinical, radiological and functional parameters. Methods: The indication of treatment for different patients was based on their clinical and neurological situation. All patients were treated with the same surgical technique. The minimal surgical follow-up was of six months. Results: Ten patients didn't have neurological problems and seven patients had some kind of neurological lesion. In the radiological evaluation during the ambulatory follow-up, all patient presented signs of consolidated spine fusion. All the patients received a grade between excellent and fair according to Odom's criteria, 13 patients presented 20\% of limitation with regard to the Neck Pain Disability Index. There was one
\end{abstract}

\section{RESUMEN}

Objetivo: Habían sido evaluados 17 pacientes que llevaban trauma de la columna cervical (C3 el C7) tratada en un período a dos años. Los pacientes con parámetros radiológicos, clínicos y funcionales fueron evaluados. Métodos: La indicación del tratamiento usado en cada paciente fue basada en el cuadro clínico y neurológico. Los pacientes fueron tratados por la misma técnica quirúrgica. El perseguir postoperatorio mínimo fuera de seis meses. Resultados: Diez pacientes no presentaron ningún comprometimento neurológico y siete pacientes presentaron un cierto grado de acometimento neurológico. En la evaluación radiográfica, los pacientes habian presentado señales de la consolidación de artrodese. Todos los pacientes estaban relacionados entre los niveles excelentes y regular de acuerdo con los criterios de Odom, 13 pacientes habian presentado el 20\% de limitación para el cuestionario Neck Pain Disability

\footnotetext{
Trabalho realizado no Hospital Universitário da Faculdade de Medicina de Uberlândia, Universidade Federal de Uberlândia - UFU - Uberlândia (MG), Brasil.

'Médico do Departamento de Ortopedia e Traumatologia da Universidade Federal de Uberlândia - UFU - Uberlândia (MG), Brasil.

${ }^{2}$ Professor do Departamento de Ortopedia e Traumatologia, chefe do grupo de coluna da Universidade Federal de Uberlândia - UFU - Uberlândia (MG), Brasil.

${ }^{3}$ Professor Titular do Departamento de Ortopedia e Traumatologia da Universidade Federal de Uberlândia - UFU - Uberlândia (MG), Brasil.

${ }^{4}$ Médico Residente do Departamento de Ortopedia e Traumatologia da Universidade Federal de Uberlândia - UFU - Uberlândia (MG), Brasil.
} 
se uma complicação de falha no implante para artrodese anterior com um ano e meio de evolução. Conclusão: $\mathrm{O}$ tratamento da fratura-luxação da coluna cervical iniciado o mais rápido possível, pela técnica de descompressão do nível lesado e redução aberta cirúrgica anterior, é um método efetivo, com menor morbidade e com um baixo grau de complicações.

\section{DESCRITORES: Vértebras} cervicais/lesões; Vértebras cervicais/cirurgia; Fraturas da coluna vertebral/diagnóstico; Fraturas da coluna vertebral/ radiografia; Resultado de tratamento complication: implant failure in an anterior fusion with 18 months of follow-up. Conclusion: The anterior approach as quickly as possible is a good option for the treatment of cervical fractures and dislocations. This approach is effective, presents low morbidity and is associated with a low number of complications.

KEYWORDS: Cervical vertebrae/ injuries; Cervical vertebrae/ surgery; Spinal fractures/ diagnosis; Spinal fractures/ radiography; Treatment outcome
Index. Una complicación ocurriera: falta del implante en una fusión anterior con 18 meses de perseguir. Conclusiones: La cirugía anterior, lo más rápidamente posible, es una buena opción en el tratamiento de las fracturas cervicales y luxaciones. Debido a que este enfoque es eficaz, con una baja morbilidad y se asocia con un bajo número de complicaciones.

DESCRIPTORES: Vértebras cervicales/lesiones; Vértebras cervicales/cirugía; Fracturas de la columna vertebral / diagnóstico; Fracturas de la columna vertebral / radiografía; Resultado del tratamiento

\section{INTRODUÇÃO}

As fraturas da coluna cervical correspondem a quase um terço de todas as fraturas da coluna vertebral. As luxações e as fraturas-luxações da coluna cervical subaxial necessitam de tratamento cirúrgico, e a literatura é clara nesse aspecto $^{1}$. A classificação mais conhecida para as lesões entre C3 e C7 da coluna cervical é a classificação de Allen e Ferguson (flexão compressão, compressão vertical, extensão compressão, flexão distração, extensão distração, flexão lateral) ${ }^{2}$. Outra classificação bastante usada, também baseada no mecanismo da fratura, é a do grupo AO, com três conjuntos principais de fraturas (A - compressão, B distração, C - rotação) $)^{3}$.

Ainda não existe um consenso sobre a melhor forma de tratamento cirúrgico para essas fraturas. Muitos centros usam a tração cervical prévia para a redução fechada da luxação cervical e, num segundo momento, a fixação da lesão cervical ${ }^{4}$. Outros preferem a redução aberta da luxação por via anterior, através da discectomia e por manobras com o distrator de Caspar e/ou um afastador interlaminar ${ }^{5}$.

A redução das luxações cervicais é mais fácil pela via posterior, mas essa técnica apresenta o risco de uma possível lesão medular pelo conteúdo do disco intervertebral lesionado. Em decorrência disso, muitos autores sugerem uma ressonância da coluna cervical ou uma discectomia no nível luxado antes que se realizem manobras para a redução da luxação cervical por via posterior ${ }^{6}$.

O tratamento precoce da lesão medular incompleta da coluna cervical é de fundamental importância para a melhor recuperação do lesado medular, sendo assim, técnicas com descompressão medular, associada à redução e artrodese ao mesmo tempo são mais efetivas e seguras para os pacientes com lesão medular incompleta.
O objetivo deste trabalho foi avaliar radiológica e funcionalmente os pacientes com fratura luxação da coluna subaxial tratados com a mesma técnica cirúrgica entre os anos de 2006 e 2008.

\section{MÉTODOS}

Dezessete pacientes com fratura da região subaxial da coluna cervical foram avaliados. Todas as fraturas foram classificadas pela classificação de Allen e Ferguson e pela classificação AO. Pacientes com fratura de mais de um segmento da coluna cervical ou com outras fraturas associadas não foram considerados neste trabalho. Os pacientes foram acompanhados funcional, clínica (nível neurológico, dor cervical) e radiologicamente (alinhamento vertebral, evidência de fusão) por um tempo médio de um ano de pós-operatório, com variação de seis meses a um ano e seis meses. Dos 17 pacientes, 2 eram do sexo feminino e 15 do sexo masculino. Com relação ao mecanismo do trauma, 12 pacientes eram vítimas de acidentes de trânsito, 2 eram vítimas de mergulho em água rasa, 2 eram vítimas de queda de altura, 1 era vítima de espancamento. Com relação ao acometimento neurológico, 4 pacientes apresentavam lesão medular completa, 3 apresentavam lesão medular incompleta e 10 pacientes não apresentavam acometimento neurológico.

O tempo médio entre a internação e a cirurgia foi de 48 horas. Todos os pacientes com fratura e ou luxação da coluna subaxial foram tratados com a mesma técnica cirúrgica.

Realizou-se um acesso retrofaríngeo à esquerda, sem tração cervical prévia, para a discectomia e descompressão medular anterior. Em seguida, tentou-se a redução das luxações com o distrator de Caspar, e a artrodese anterior 
com placa. Nos casos em que não foi alcançada a redução por via anterior, foi realizado um acesso posterior para a redução da luxação e fixação posterior, e em seguida a artrodese anterior (Figura 1). Todos os pacientes foram avaliados funcionalmente pelos critérios de Odom's e o questionário Neck Pain Disability Index ${ }^{5,7}$.

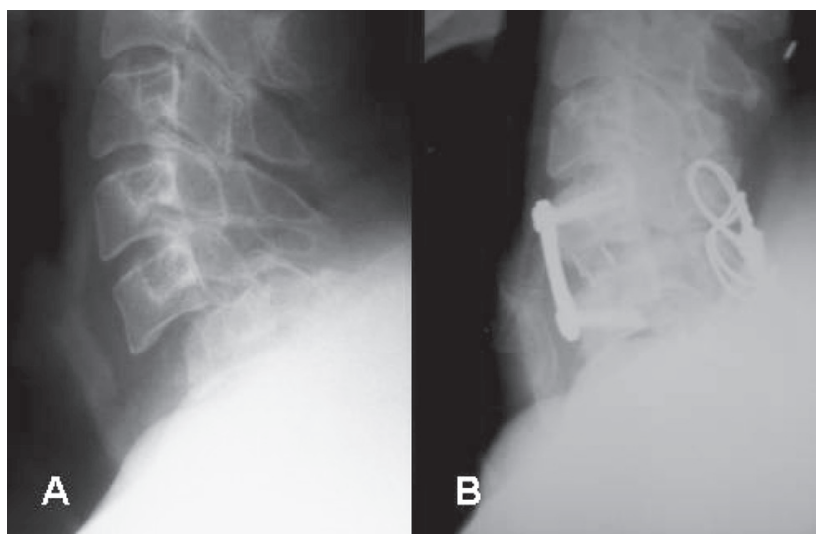

Figura 1

Radiografia em perfil do paciente com lesão neurológica incompleta e luxação facetária bilateral (A). Radiografia em perfil do pós-operatório imediato, sendo que nesse paciente não foi alcançada uma redução satisfatória pela via anterior inicialmente, necessitando-se uma tripla via (B).

\section{RESULTADOS}

A Tabela 1 apresenta a vértebra fraturada, as classificações utilizadas e o tratamento realizado em cada paciente.
Em dois pacientes, a redução não foi alcançada pela via anterior, sendo necessária a via posterior para a redução da lesão. Um paciente apresentava luxação unilateral com cominuição facetária, e outro apresentava uma luxação facetária bilateral com fratura de um dos pedículos vertebrais. No terceiro paciente, tratado com a artrodese combinada, a fixação posterior foi realizada em um segundo momento, após a redução e artrodese anterior, porque o paciente apresentava uma lesão óssea e ligamentar extensa da transição cervicotorácica.

$\mathrm{Na}$ avaliação radiográfica, 14 pacientes apresentaram sinais de consolidação óssea entre os corpos vertebrais. Dois pacientes não apresentavam sinais de consolidação com um ano de evolução, existia uma interlinha entre a parte superior do enxerto e o corpo vertebral, mas esse achado radiográfico não se relacionou a manifestações clínicas e funcionais insatisfatórias. Um dos pacientes apresentou um desalinhamento entre os corpos vertebrais menor que $3 \mathrm{~mm}$ após a redução e artrodese anterior, que não se modificou durante as radiografias dinâmicas em flexão e em extensão no acompanhamento pós-operatório; da mesma forma, esse desalinhamento não foi relacionado a avaliações clínicas e funcionais ruins.

$\mathrm{Na}$ avaliação clínica, nenhum paciente apresentou piora do nível neurológico após o tratamento cirúrgico da fratura da coluna cervical. Um paciente com lesão cervical incompleta Frankel C evoluiu para Frankel D no decorrer do pós-operatório. Um paciente apresentou como complicação uma soltura do material de síntese com a artrodese já consolidada com um ano e meio de pós-operatório, sendo retirado o material de síntese. Na avaliação funcional pelos

\section{TABELA 1 - Características das fraturas dos pacientes e tratamento utilizado}

\begin{tabular}{lcccc}
\hline Iniciais paciente & Vertebra fraturada & Classificação AO & Classificação Allen & Tratamento \\
\hline JNS & C5 & C3 & Flexão lateral & Artrodese anterior \\
ECM & C6 & B2 & Flexão compressão & Artrodese anterior \\
FJT & C4 & C3 & Flexão lateral & Artrodese anterior \\
FPS & C6 & B1 & Flexão compressão & Artrodese anterior \\
QWF & C6 & B1 & Flexão compressão & Artrodese combinada \\
IVL & C7 & B1 & Flexão compressão & Artrodese combinada \\
JOQ & C5 & C3 & Flexão lateral & Artrodese anterior \\
MN & C5 & B1 & Flexão compressão & Artrodese combinada \\
$M L A$ & C4 & B1 & Flexão compressão & Artrodese anterior \\
$M S T$ & C6 & C3 & Flexão lateral & Artrodese anterior \\
MJB & B1 & Flexão compressão & Artrodese anterior \\
NQO & C5 & B1 & Flexão compressão & Artrodese anterior \\
PSVB & C5 & B1 & Flexão compressão & Artrodese anterior \\
UQS & C4 & B1 & Flexão compressão & Artrodese anterior \\
VBS & C4 & B1 & Flexão compressão & Artrodese anterior \\
VSB & C5 & C2 & Flexão lateral & Artrodese anterior \\
LM & C3 & B1 & Flexão compressão & Artrodese anterior \\
\hline
\end{tabular}


critérios de Odom, 10\% referiram que o resultado do tratamento foi excelente, $60 \%$ consideraram bom, $20 \%$ regular e $10 \%$ ruim. Segundo o questionário Neck Pain Disability Index, 13 pacientes apresentaram 0 a $20 \%$ de limitação (dificuldade para ficar sentado ou de pé por tempo prolongado, mas os pacientes não fazem uso de medicação para dor), 2 pacientes apresentaram entre 20 e $40 \%$ de limitação (dor mais acentuada; não conseguiram voltar ao trabalho, apresentaram limitações na vida social), 1 paciente apresentou entre 40 e $60 \%$ (dor limitante, dificuldades com o sono, cuidados pessoais) e 1 paciente apresentou limitação entre 60 e $80 \%$ (dor limitante em todos os aspectos para o paciente na vida social e no trabalho).

\section{DISCUSSÃO}

A tração cervical para a redução fechada da luxação subaxial é um método bastante utilizado ${ }^{6}$. Esta técnica pode alcançar a redução na maioria dos casos e é um arsenal terapêutico muito importante para descompressão medular na emergência ${ }^{8}$. No presente trabalho, não foi utilizada porque todos os pacientes com lesão neurológica completa ou lesão incompleta puderam ser operados emergencialmente no máximo dois dias após o trauma (Figura 2). Ao mesmo tempo, a discectomia anterior no nível luxado descomprime o canal, diminuindo os riscos de comprometimento neurológico e minimizando a necessidade da ressonância para avaliar a lesão discal na emergência, fator importan-

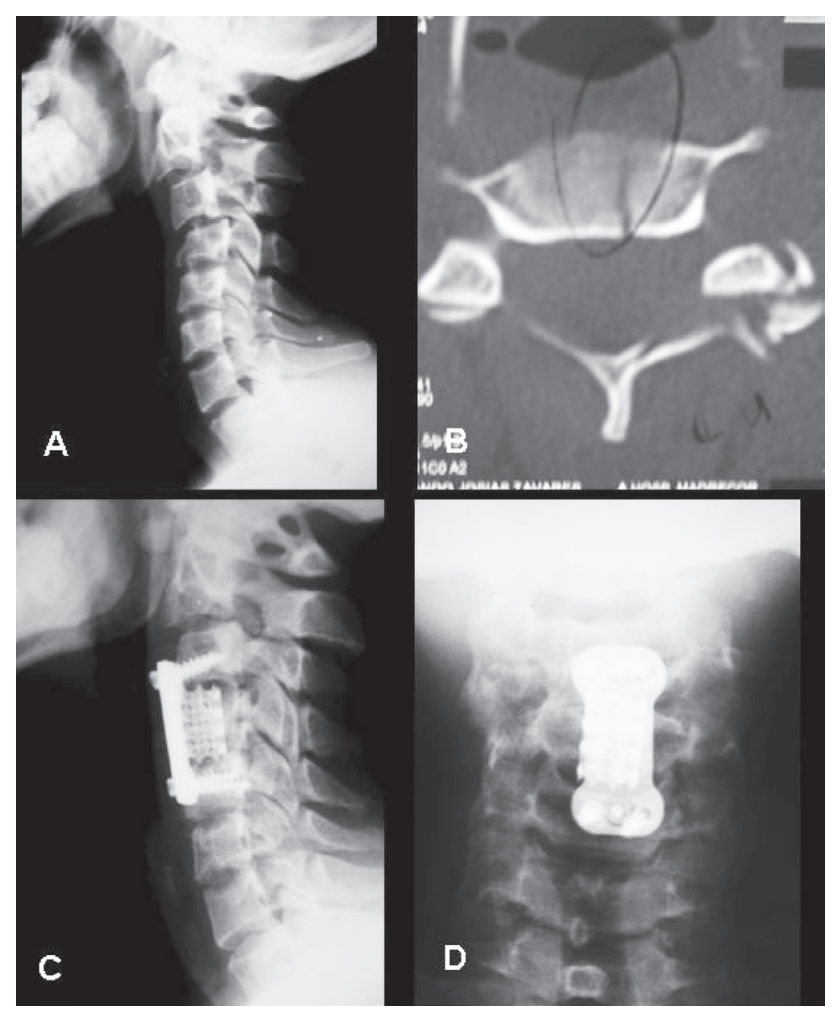

Figura 2

Radiografia e tomografia de um paciente com fratura de C4 e fratura luxação unifacetária C3-C4 (A e B). Radiografia em anteroposterior e perfil com três meses de pós-operatório (C e D). te para muitos hospitais de urgência no mundo que não possuem ressonância disponível 24 horas na emergência. Muitas vezes, a falta desse exame na emergência impossibilita a instalação da tração cervical no paciente, pelo risco do não-diagnóstico de uma lesão discal cervical e da piora do quadro neurológico com a instalação da tração cervical em um paciente com lesão neurológica incompleta.

A redução da luxação ou da fratura luxação cervical por via anterior através do distrator de Gaspar é um método seguro e, na maioria das vezes, efetivo ${ }^{6}$. Além disso, é prático porque, em um único procedimento, é possível reduzir o desalinhamento cervical anterior e fixar a fratura (Figura 3). Em dois dos pacientes estudados não foi possível alcançar a redução pela cervicotomia anterior. O primeiro paciente apresentava uma luxação unifacetária e fratura da faceta articular cominuida; o segundo, apresentava luxação bifacetária e fratura de um dos pedículos vertebrais. Nesses dois casos foi realizada a tripla via: anterior para discectomia descompressiva, posterior para redução e fixação posterior e anterior para enxerto estrutural e fixação com placa. Segundo os critérios de Odom e o questionário Neck Pain Disability Index, 70\% dos pacientes apresentaram bons resultados. Esses achados funcionais são semelhantes aos de outro autor ${ }^{7}$. Resultados funcionais ruins estiveram relacionados ao acometimento neurológico do paciente no momento do trauma.

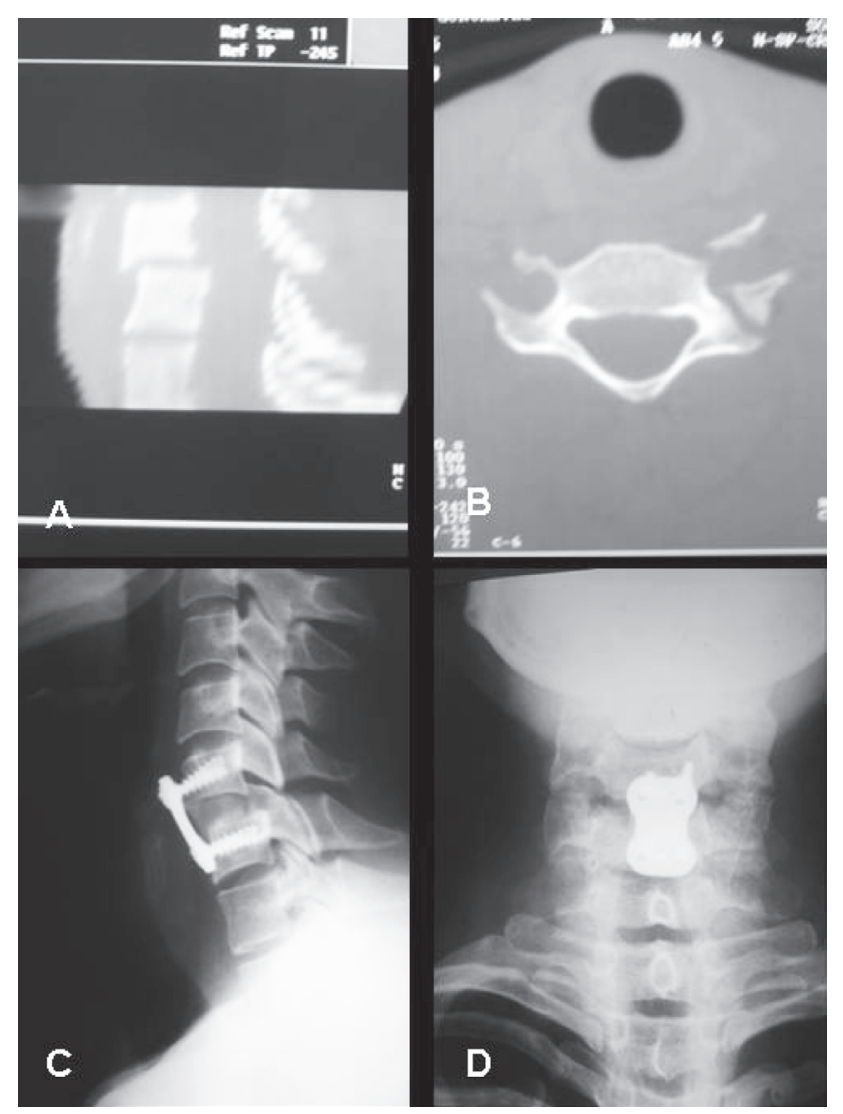

Figura 3

Tomografia de paciente com fratura luxação c5-c6 em corte sagital e axial (A e B). Radiografia em anteroposterior e perfil com seis meses de pós-operatório ( $C$ e D). 


\section{CONCLUSÕES}

A abordagem anterior, o mais cedo possível, da fratura-luxação cervical é um método que se mostrou efetivo, com uma baixa taxa de complicações e uma menor taxa de morbidade, pois evitou a tração prévia para a redução da luxação e possibilitou a descompressão rápida da medula vertebral.

\section{REFERÊNCIAS}

1. Kwon BK, Vaccaro AR, Grauer JN, Fisher CG, Dvorak MF. Subaxial cervical spine trauma. J Am Acad Orthop Surg. 2006;14(2):78-89

2. Allen BL Jr, Ferguson RL, Lehmann TR, O'Brien RP. A mechanistic classification of closed, indirect fractures and dislocations of the lower cervical spine. Spine. 1982;7(1):1-27.

3. Aebi M, Nazarian S. [Klassification der Halswirbelsäulenverletzungen]. Orthopäde. 1987;16:27-36. German.

4. Brodke DS, Anderson PA, Newell DW, Grady MS, Chapman JR. Comparison of anterior and posterior approaches in cervical spinal cord injuries. J Spinal Disord Tech. 2003;16(3):229-35.
5. Defino HA, Figueira FG, Camargo LS, Canto FR. Tratamento das lesões traumáticas da coluna cervical por meio da abordagem anterior. Acta Ortop Bras. 2007;15(1):30-4.

6. Reindl R, Ouellet J, Harvey EJ, Berry $\mathrm{G}$, Arlet V. Anterior reduction for cervical spine dislocation. Spine. 2006;31(6):648-52.

7. Kandziora F, Pflugmacher R, Scholz M, Schnake K, Putzier M, Khodadadyan-Klostermann C, et al. Treatment of traumatic cervical spine instability with interbody fusion cages: a prospective controlled study with a 2-year follow-up. Injury. 2005;36 Suppl 2:B27-35.
8. Darsaut TE, Ashforth R, Bhargava R, Broad R, Emery D, Kortbeek F, et al. A pilot study of magnetic resonance imaging-guided closed reduction of cervical spine fractures. Spine. 2006;31(18):2085-90.

\section{Correspondência}

Fabiano Ricardo de Tavares Canto

Departamento de Ortopedia e Traumatologia da Universidade Federal de Uberlândia

Avenida Pará, 1720

CEP 38405-320 - Uberlândia (MG), Brasil

Tel./Fax: (34) 3218-2202

E-mail: ftcanto@hotmail.com 